\section{Prevención Del Embarazo Precoz en estudiantes de colegios fiscales en} Ecuador

Pregnancy Pregnancy Prevention in fiscal

school students in Ecuador

\section{Elizabeth Briggitte Quijije}

Logroño

Universidad de Guayaquil

Facultad de Filosofia

Letras y Ciencias de la

Educacion

https://orcid.org/0000-

0001-6981-8220

elizabethquijije19962@g

mail.com

Guayaquil - Ecuador

http://www.jah-

journal.com/index.php/jah

Journal of American health

July - December vol. 2. Num.

$1-2019$

Esta obra está bajo una

Licencia Creative Commons

Atribución-NoComercial-

CompartirIgual 4.0

Internacional.

RECIBIDO: 15 DE MARZO 2018

ACEPTADO: 1 DE JULIO 2018

PUBLICADO: 4 DE ENERO 2019

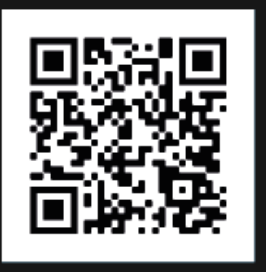

Scan this QR

code with your

smart phone or

mobile device to

read more papers

\section{RESUMEN}

Antecedentes. - El inicio de la actividad sexual en adolescentes, está asociada a pobre planificación familiar, mayor tasa de fecundidad y riesgo de adquisición de enfermedades de transmisión sexual, además del aumento de embarazos adolescente. Objetivos. - Este trabajo tuvo como objetivo general determinar un perfil clínico - epidemiológico del embarazo en la adolescencia relacionado con antecedentes familiares en el hospital General Villamil Playas mediante el análisis de las características sociales, culturales y médicas con el fin de proponer un programa de educación en salud, este objetivo puede ser alcanzado al identificar las características de las pacientes, establecer factores de riesgo de embarazo adolescente considerando los antecedentes familiares y proponer un programa de educación para la salud dirigido a hombres y mujeres adolescentes. Metodología. - Se trata de una investigación cuantitativa, descriptiva con un diseño no experimental, prospectiva y de corte transversal donde se incluyeron mujeres adolescentes embarazadas que asistieron a la consulta en el área de ginecología del Hospital de General Villamil Playas durante el primer semestre del 2016, que en total fueron 352 y se tomó una muestra de 100 pacientes. Resultados. - El $85 \%$ de las madres adolescentes presentaron antecedentes maternos de embarazo precoz, con una marcada diferencia estadística al aplicar la prueba de $\mathrm{CHI}$ cuadrado. Conclusión. -Entre los principales factores de riesgo se observa una comunicación deficiente entre los padres y sus hijos sobre sexualidad, debido a que la mayoría aprendieron sobre este tema de parte de sus amigos y no de sus padres o la 
escuela, además en algunos casos no se sabía del noviazgo de sus hijas.

PALABRAS CLAVE: Embarazo adolescente, Antecedentes maternos, Programa educativa de prevención.

\section{ABSTRACT}

Background. - The onset of sexual activity in adolescents is associated with poor family planning, higher fertility rate and risk of acquiring sexually transmitted diseases, in addition to the increase in adolescent pregnancies. Objectives. - This study aims to determine the clinical and epidemiological profile of adolescent pregnancy related to family history in General Villamil Beaches hospital by analyzing social, cultural and medical characteristics in order to propose a health education program, This objective can be achieved by identifying the characteristics of The patients, establishing risk factors for adolescent pregnancy by considering the family history and proposing the health education program aimed at adolescent men and women. Methodology. - This is a quantitative, descriptive research with a non-experimental, prospective and crosssectional design that included pregnant women who attended the consultation in the gynecology area of General Villamil Playas Hospital during the first half of 2016, which In total There were 352 and a sample of 100 patients was taken. Results. - 85\% of adolescent mothers had a maternal history of precocious pregnancy, with a marked statistical difference when applying the $\mathrm{CHI}$ square test. $(P<0.001)$ Conclusion. - Among the main risk factors are poor communication between parents and their children about sexuality, because most of them learned about this from their friends, not from their parents or the school. Knew of the courtship of his daughters.

KEY WORD: Adolescent pregnancy, Maternal history, Prevention educational program

\section{INTRODUCCIÓN}

El embarazo en la adolescencia es ya un problema social, económico, salud pública considerable magnitud, tanto como para los jóvenes padres hijos familia y el ambiente cual los rodea.

Consideramos que el número de embarazos en adolescentes sigue siendo elevado según el estudio realizado por el banco mundial. El ecuador como el sexto país con la tasa más alta de embarazo en adolescentes en América Latina y el caribe, la actividad sexual cada vez comienza a una edad más temprana actualmente se maneja el dato d los 12 años, entonces es necesario preguntarnos y empezar a investigar porque los jóvenes deciden tener relaciones sexuales.

Dentro del grado de embarazo los adolescentes que se encontraban estudiando el $80 \%$ de varones y mujeres si continúan con sus estudios, pero la mayoría de estos jóvenes ya no tienen las mismas metas que cuando no tenían una responsabilidad como la de ser padres por la cual se centran a estudiar por el simple motivo de que él bebe está en camino la cual es la razón de que el promedio estudiantil sea afectado y ahí se producirá la llamada deserción escolar (abandono escolar). Esto nos incentiva a empezar una plática con los padres sin titubees, ni miedos, y buscar métodos con ayuda del docente facilitador con palabras y métodos adecuados para que nosotros los adolescentes podamos captar el mensaje de una forma cierta y concisa. 
La falta de información y el desconocimiento sobre el uso de los anticonceptivos, la poca accesibilidad a los servicios de salud por barreras socioculturales, económicos y educación inadecuada entre padres e hijos contribuyen al inicio temprano de las relaciones sexuales, generando abortos deserción escolar (abandono escolar), embarazo precoz, infecciones de transmisión sexual.

Las probabilidades de que las niñas de 15 y 19 años mueran durante el parto duplican con las de las mujeres con más de 20 años, este riesgo es 5 veces mayor en el caso de las niñas menores de 15 años.

El objeto de la investigación lo conforman las características clínicas - epidemiológicas del embarazo en la adolescencia, que se relacionan con una serie de factores personales, familiares y culturales, mientras que el campo de estudio es la sexualidad y ginecología en la adolescencia, que implica un reconocimiento amplio de aquellos aspectos relacionados con el inicio de las relaciones sexuales y que pueden llevar a una posible solución a esta problemática.

El embarazo precoz es un mal que cada vez más se está extendiendo en las sociedades desarrolladas. Hasta no hace mucho, se pensaba que este tipo de embarazos sólo se producían en los países del tercer mundo, pero en los últimos años, con la libertad sexual que ha venido dándose en las sociedades del primer mundo, cada vez más son las adolescentes que quedan en cinta antes de estar completamente desarrolladas.

Un embarazo precoz es aquél que sufre una adolescente que ya está en edad fértil, pero que no está completamente formada ni está preparada todavía para ser madre. En este sentido, se suele considerar que las mujeres de menos de dieciocho años embarazadas están sufriendo un embarazo precoz, mientras que las de diecinueve o más, tienen un embarazo normal.

Esta distinción es relativa, puesto que cada mujer se desarrolla de diferente forma y en distinto tiempo. Sin embargo, sí es cierto que la mayoría de adolescentes no están preparadas para ser madres, y también es cierto que las mujeres de esta edad que quedan embarazas, suelen sufrir un embarazo de alto riesgo, con graves consecuencias tanto para ellas como para los pequeños. La adolescencia es un periodo del desarrollo biológico, psicológico, sexual y social inmediatamente posterior a la niñez y que comienza con la pubertad. Es un periodo vital entre la pubertad y la edad adulta, su rango de duración varía según las diferentes fuentes y opiniones médicas, científicas y psicológicas, generalmente se enmarca su inicio entre los 10 y 12 años, y su finalización a los 19 o 24.1

Anteriormente, la Organización Mundial de la Salud consideraba a adolescencia como el período comprendido entre los 10 y 19 años, comprendida dentro del período de la juventud -entre los 10 y los 24 años-. La pubertad o adolescencia inicial es la primera fase, comienza normalmente a los 10 años en las niñas y a los 11 en los niños y llega hasta los 14-15 años. La adolescencia media y tardía se extiende, hasta los 19 años. A la adolescencia le sigue la juventud plena, desde los 20 hasta los 24 años.

\section{MATERIALES Y MÉTODOS}

Esta investigación tiene un enfoque cuantitativo, haciendo énfasis en una investigación descriptiva con un diseño no experimental, prospectiva y de corte transversal, donde se han analizado las características de las pacientes, de allí el enfoque descriptivo, además de hacer 
un análisis más profundo de las características que se consideran factores de riesgo con relación directa de los antecedentes maternos de embarazo precoz.

Entre los métodos teóricos se aplicó el analítico, ya que la información fue recopilada directamente de las pacientes en unidades pequeñas (Variables) luego estas fueron integradas en un solo conjunto dando paso a las estrategias deductivas. Entre los métodos empíricos se utilizó una hoja de recolección de datos y una encuesta para determinar algunos factores relacionados con el embarazo adolecentes, donde es importante considerar que los datos se recogieron directamente de las historias clínicas, así y la encuesta arrojó información relacionada con del embarazo precoz con antecedentes familiares, específicamente los maternos

El embarazo adolescente está relacionado directamente con antecedentes maternos de embarazo precoz, que parten de una buena comunicación, por lo que es necesario una intervención educativa.

\section{RESULTADOS}

ESTE proyecto se realiza pensando en los estudiantes de la unidad educativa PATRIA ECUATORIANA de la ciudad de GUAYAQUIL para que ellos sean beneficiados y para que tomen conciencia que la sexualidad es una etapa que hay que tomarla con responsabilidad y de una manera segura, los jóvenes tienen que ser orientados para que no sean juzgados por el medio que los rodea y dar a conocer que hay muchas alternativas para no quedar embarazadas a temprana edad.

Para prevenir muchos embarazos en adolescentes estamos preparando esta prevención para que los adolescentes tengan una mejora en sus decisiones y puedan continuar en sus estudios y no tengan un stop en el transcurso de su año lectivo ya que tener a un bebe siendo menores de edad y les ocasionara muchas dificultades para culminar los estudios y concentrarse con toda la totalidad necesaria para nuestra educación.

Esta prevención es para que los adolescentes tengan una información adecuada de la sexualidad a base de hechos, experiencias, sucesos e investigaciones para que ellos tomen conciencia y sus decisiones no sean erróneas ya que si es un adolecente que mantiene relaciones sexuales deberá tener la debida información de los anticonceptivos y los métodos para no tener un embarazo precoz o no deseado.

Los adolescentes necesitan tener información sobre los método anticonceptivos, la fertilidad , las muertes que pueden causar las enfermedades de transmisión sexual y el riesgo que corren las adolescentes y él bebe en el parto ya como antes mencionado las adolescentes no tienen la capacidad para llevar un embarazo normal y sin riesgos como una joven de 20 o más edad.

La mayor razón para esta prevención es que en los últimos años ha sido alarmante el gran aumento del porcentaje de embarazos en adolescentes y muchos de los adolescentes no culminan sus estudios por el solo hecho de que serán padres y tendrán otras obligaciones por eso es qué dicen “... TODO A SU DEBIDO TIEMPO “.. ya que al tener al bebe el padre tendrá que trabajar para poder sostener un hogar y la madre tendrá que estar pendiente del bebe en camino, esto no quiere decir que todos aquellos adolecentes que tienen un embarazo precoz no podrán culminar sus estudios podrán si pero habrán muchos obstáculos, la cual era más fácil si ese embarazo precoz no se presenta con sus debidas precauciones ya que existen muchos métodos anticonceptivos. 
Actualmente en nuestra sociedad existen muchos casos de adolescentes embarazadas las cuales hacen que el estilo de vida del adolescente lleve un ritmo diferente en el cual en algunos casos abandonan sus estudios y por ende afecta al adolescente a la sociedad y a su economía familiar y como para el país; que quiero decir con esto, si no logramos culminar los estudios no podremos ser un profesional ya que hay un porcentaje elevado de tantas madres solteras sin culminar sus estudios porque tienen que trabajar para poder sustentar a sus hijos y esto ocasiona otro problema para la sociedad.

Nuestro proyecto se está realizando en nuestra institución educativa PATRIA ECUATORIANA en las jornadas matutina y vespertina ya sea dentro de las aulas de clases en el patio central o en la sala de audiovisual ; en cual este proyecto es un proceso de tres meses en el cual vamos a ir indagando y realizando las actividades necesarias para nuestro proyecto de prevención del embarazo precoz en adolescentes, vamos a dar a conocer nuestro proyecto mediante charlas, actividades llamativas, entregando trípticos que obtengan la información necesaria para la prevención, también nos ayudaremos con objetos visuales como carteleras con el mensaje deseado .

Contamos con la predisposición de los docentes, la creatividad de cada uno de nuestros integrantes de grupo, el servicio de cada compañero y de los docentes facilitadores, también cuento con los sitios web y los textos educativos.

La utilidad de este proyecto es tomar en cuenta la orientación, guía, soporte y el conocimiento para todos los adolescentes de nuestra institución del significado de todos los puntos que tratemos a dar a conocer como el cual tenemos como primer punto:

- La responsabilidad -. al tener un embarazo precoz sin los conocimientos adecuados tanto como en la salud del adolecente, la educación, entre la sociedad y la economía.

- Reducir la incidencia de nuevos casos.

- Recomendar. _acciones para reducir el embarazo en estudiantes de nuestra institución.

Es importante también reconocer los factores y causas que aumentan el riesgo de embarazos y por lo tanto estos factores no son beneficio para los adolescentes, padres de familias y el ambiente que los rodea, también peligra la vida de la adolecente embarazada ya que su organismo tiene implicaciones muy serias ya que no está apto para tener un embarazo en esta etapa de la vida que es la DOLESCENCIA , la información y el apoyo de parte de familiares como lo son sus padres y el entorno en el cual se rodean los adolescentes sea adecuada referente a las relaciones sexuales, las enfermedades tanto de transmisión sexual como otras enfermedades que puedan presentar los adolescentes y los métodos anticonceptivos estos son algunas problemáticas que desde casa nos pueden orientar .

Tenemos como beneficio principal para los estudiantes de nuestra institución educativa dar a conocer la información sobre el embarazo precoz, la sexualidad, enfermedades de transmisión sexual, la planificación familiar y sobre los anticonceptivos un punto muy importante que muchos adolescentes no tienen el conocimiento adecuado ; también nos beneficia en un mejor futuro para nuestro país ya que nuestra finalidad es llegar a que los adolescentes tenga una mejor formación tanto como personas y profesionales la conservación de nuestras culturas .

La calidad de nuestro proyecto puede incentivar a otros estudiantes para que continúen combatiendo esta problemática por el cual podremos llegar a tener un mejor resultado general ya no tan solo en nuestra institución educativa sino que fuera de este campo muy importante 
aunque es muy favorable en las instituciones porque contamos con la colaboración de muchos adolescentes y asi puede llegar un mejor mensaje de esta prevención para mejorar nuestra sociedad y recuperar la educación y las culturas que se van perdiendo .

También realizaremos charlas para los padres para que ellos también tengan una información adecuada y correcta para poder aconsejar a sus hijos porque muy bien sabemos que siempre el ejemplo viene desde casa y que en las instituciones educativas nos ayudan a tener un concepto más preciso y correcto.

a. General- disminuir el embarazo precoz en las adolescentes de nuestra institución " patria ecuatoriana" haciendo charlas para que las alumnas tengan conocimiento de lo que es un embarazo precoz, y porque es que hay que prevenir los embarazos en las adolescentes, ya que traer un bebe al mundo y a una temprana edad tiene el riesgo de que el bebe o la mamá fallezca, a la vez unos padres adolescentes tienen la consecuencia de dejar los estudios porque tiene que dedicarse a trabajar para darle un futuro mejor a su hogar.

b.Especifico.- Nuestro objetivo específico es realizar charlas y poder hacer llegar nuestro temas a las(o) jóvenes adolescentes de nuestra institución para que tengan un conocimiento e información de lo que es un embarazo precoz.

\section{DISCUSIÓN}

Los adolescentes empiezan con una vida sexual desde muy temprana edad y se enfrentan a riesgos serios como embarazos no deseados, para luego empezar un ciclo de pobreza, ya que no terminan sus estudios al dedicarse a las tareas del hogar, además un porcentaje alto continua a vivir con sus padres o los padres de su cónyuge por lo que aumentan las dificultades económicas y afectivas de no tener independencia, además en muchos casos el cónyuge es un estudiante en las mismas condiciones de ella y en otros es alguien mayor por lo que se aprecia un grado de dependencia emocional.

\section{REFERENCIAS}

1. Cobankara F, Unlü N, Altinöz H, Füsun O. Effect of home bleaching agents on the roughness and surface morphology of human enamel and dentine. International Dental Journal. 2004;: p. 211-218.

2. Götz H, Duschner H, White D, Klukowska M. Effects of elevated hydrogen peroxide 'strip' bleaching on surface and subsurface enamel including subsurface histomorphology, microchemical composition and fluorescence changes. Journal of Dentistry. 2007;: p. 457- 466.

3. Kimyai S, Oskoee S, Rafighi A, Valizadeh H, Ajami A, Helali Z. Comparison of the effect of hydrogel and solution forms of sodium ascorbate on orthodontic bracket enamel An in vitro study. Indian J Dent Res. 2010;: p. 21: 54-58. 
4. Sung E, Chan S, Mito R, Caputo A. Effect of carbamide peroxide bleaching on the shear bond strengthof composite to dental bonding agent enhanced enamel. J Prosthet Dent. 1999;: p. 595-599.

5. Homewood C, Tyas MW. Bonding to previously bleached teeth. Aust Orthod J. 2001;: p. 2734.

6. Segarra E, Espasa SdDE, Boj-Quesada JR. Influencia de diferentes sistemas de profilaxis en la adhesión de un sellador de fisuras al esmalte. RCOE. 2005;: p. 177-182.

7. Demarco F, Freitas J, Silva M, Justino L. Microleakage in endodontically treated teeth: influence of calcium hydroxide dressing following bleaching. Int Endod J. 2001;: p. 495 - 500. 